\title{
Autocatalytic models describing ruminal in situ digestion
}

\author{
$J$ Van Milgen \\ INRA, Station de Recherche sur la Nutrition des Herbivores, Theix, \\ 63122 Saint-Genès-Champanelle, France
}

\begin{abstract}
Digestion of particulate matter in the rumen is a microbial enzymatic process and probably depends on both substrate and enzyme availability. Although microbes can be considered the cause of digestion, they are also the result of digestion, being one of the digestion end-products. Thus, digestion partially is an autocatalytic process.
\end{abstract}

In the classical digestion model (model 1), rate of digestion ( $d S / d t$ is proportional only to the concentration of potentially digestible substrate (S). The effect of other factors affecting digestion is represented by the fractional digestion rate, which is assumed to be constant $\left(k_{\mathrm{d}}\right)$. To account for time-varying effects of microbes (or enzymes) on digestion of substrate, 2 models are proposed which are based on a non-constant fractional digestion rate $(Z)$, such that $d S / d t=-Z S$ :

1. $\mathrm{d} Z / \mathrm{d} t=0 ; Z=0$ for $t \leq t_{4}$ and $Z=k_{\mathrm{d}}$ for $t>4$ 2. $\mathrm{d} Z \mathrm{~d} d t=\left(Z_{\mathrm{i}}-Z\right) Z$ with $Z_{0} \neq 0 ; Z=Z_{\mathrm{i}} Z_{0} /\left(Z_{0}+\right.$ $\left(Z_{i}-Z_{0}\right) \exp \left(-Z_{i} \theta\right)$

3. $\mathrm{d} Z / \mathrm{d} t=\left(Z_{\mathrm{i}}-Z\right) k_{\mathrm{z}}$ with $Z_{0}=0 ; Z=Z_{\mathrm{i}}(1-$ $\exp \left(-k_{z} t\right)$

which yield for $Y$, the total quantity of substrate remaining $\left(\mathrm{Stt}_{\mathrm{i}}\right)$ :
1. $Y=t_{\mathrm{d}}+t_{\mathrm{i}}$ for $t \leq t_{;} ; S=t_{\mathrm{d}} \exp \left(-k_{\mathrm{d}}\left(t-t_{\mathrm{i}}\right)\right)+f_{\mathrm{i}}$ for $t>t$
2. $Y=f_{d} Z_{i}\left(Z_{0} \exp \left(Z_{i} t\right)+Z_{i}-Z_{0}\right)+f_{i}$
3. $Y=f_{\mathrm{d}} \exp \left(Z_{\mathrm{i}}\left(1-k_{\mathrm{z}} t-\exp \left(-k_{\mathrm{z}} t\right)\right) / k_{\mathrm{z}}\right)+f_{\mathrm{i}}$

where $t$ is the incubation time $(h), t_{d}$ the potentially digestible fraction $(g / g), f_{i}$ the indigestible fraction $(g / g), k_{d}$ the fractional digestion rate constant $(1 / h), t$ the discrete lag time $(h), Z_{0}$ the initial value of $Z(1 / \mathrm{h}), Z_{\mathrm{i}}$ the asymptotic value of $Z$ $(1 / \mathrm{h})$, and $k_{\mathrm{z}}$ the fractional rate of change of $Z$ $(1 / h)$. The models were evaluated by fitting the equations to in situ data of 4 roughages.
Microbes involved in digestion of substrate are either synthesized within the system (in situ bag) or originate from the environment (rumen fluid surrounding in situ bags). Model 2 ( $Y$ is the logistic function) is based on the conversion of substrate into microbes (or enzymes), but does not account for exchange of microbes between the system and its environment. Model 3 assumes that the effect of microbes on digestion is timedependent. Although it does not explicitly account for the origin of microbial mass, it has properties that cannot be included in model $1\left(e g, Z_{0}=0\right)$.

The residual standard deviation (RSD) and estimates for $t_{d}$ and $f_{i}$ were generally similar between models. The $Z_{i}$ (representing the fractional digestion rate after infinite incubation) for model 3 was of a similar magnitude to $k_{d}$ in model 1. However, $Z_{i}$ for model 2 was 35 to $95 \%$ higher than $k_{d}$. The $t, Z_{0}$ and $k_{z}$ are difficult to compare between models because they are based on different concepts. Parameter estimates for orchard grass hay are given in table $I$ as an example. Both models are suitable alternatives to the classical model.

Table I. Digestion kinetic parameters for orchard grass hay.

Model 1 Model $2 \quad$ Model 3

\begin{tabular}{|c|c|c|c|}
\hline $\begin{array}{l}\text { RSD }(g / g) \\
f_{d} \\
f_{i} \\
k_{d} \text { or } Z_{i}\end{array}$ & $\begin{array}{l}0.023 \\
0.597 \\
0.227 \\
0.034 \\
4.61\end{array}$ & $\begin{array}{l}0.024 \\
0.615 \\
0.237 \\
0.047\end{array}$ & $\begin{array}{l}0.021 \\
0.604 \\
0.230 \\
0.036\end{array}$ \\
\hline $\begin{array}{l}t_{1} \\
z_{0}\end{array}$ & $\begin{array}{c}4.61 \\
-\end{array}$ & $\overrightarrow{0.023}$ & $\overline{-}$ \\
\hline$k_{z}$ & - & - & 0.198 \\
\hline
\end{tabular}

